\title{
Maximum-Minimum Energy Based Spectrum Sensing under Frequency Selectivity for Cognitive Radios
}

\author{
Sener Dikmese, Paschalis C. Sofotasios, Markku Renfors and Mikko Valkama \\ Department of Electronics and Communications Engineering \\ Tampere University of Technology \\ Tampere, Finland \\ \{sener.dikmese, paschalis.sofotasios, markku.renfors and mikko.e.valkama\}@tut.fi
}

\begin{abstract}
Cognitive Radio (CR) technology which uses intelligent signal processing at the physical layer of a wireless system has been considered for coordinating better the spectral resources. In this study, we investigate spectrum sensing methods which utilize the frequency variability of the energy spectral density which is introduced by the primary transmissions. The variability is partly due to the transmitted spectrum shape and party introduced by the frequency selective multipath channel. The spectral variability is observed by dividing the sensing frequency band into relatively narrow subbands and comparing the subband energies. We compare the subband energy based methods against the eigenvalue based sensing method, which exploits the signal correlations introduced by the primary transmission. Eigenvalue based sensing is known to be robust against the inevitable noise uncertainty, which severely limits the usability of energy detection for primaries with low SNR. Our results demonstrate for the subband energy detection methods, similar robustness against noise uncertainty as the eigenvalue based methods have.
\end{abstract}

Index Terms-Cognitive radio, spectrum sensing, maximum minimum energy detection, frequency selective channel.

\section{INTRODUCTION}

Cognitive radio (CR) is emerging as an attractive solution to the challenge of spectral congestion in wireless communication systems. In CR networks, a secondary unlicensed user (SU) is allowed to use the spectrum when the primary licensed user (PU) system is idle [1], [2]. Hence, spectrum sensing sensing is an important function irrespective of the $\mathrm{CR}$ architecture and becomes critical in $\mathrm{CR}$ communication systems.

Energy detection, which is commonly formulated as a Neyman-Pearson type binary hypothesis testing problem, is modelled by the chi-square, normal or gamma type statistical distributions [1]-[5]. Energy detection is the most popular sensing method thanks to its simple practical realization and low computational complexity [3], [4]. Based on these properties, many studies have been reported in the context of Additive-White-Gaussian-Noise (AWGN), fading channels, diversity techniques and collaborative detection [6]-[11].

In practice, sensing performance is affected by the requirement of reliable operation with very low SNR of the PU signal in the presence of significant uncertainty about the actual noise power [12]. In many traditional energy detector based spectrum sensing studies, the noise variance is assumed to be known perfectly according to the previous energy measurement [10], [11]. It is clear that the performance of the traditional energy detector based sensing significantly decreases under noise uncertainty.

Spectrum sensing methods which utilize the frequency variability of the energy spectral density (ESD) within the sensing band have been investigated in [13]-[15]. Subbandwise energy detection is utilized for evaluating the ESD variability, which is assumed to be due to the frequency selective multipath channel. When the channel is frequency selective and the noise is AWGN, this sensing approach can effectively remove the noise floor, resulting in the elimination of noise uncertainty effects. The decision statistic is based on order statistics of the differential ESD [13]. This method, which is here referred to as differential Max-Min ED, was shown to be robust against the noise uncertainty with low SNR.

In this paper, we develop a novel subband energy based sensing method and investigate the performance and complexity tradeoffs in noise uncertanty tolerant spectrum sensing. More specifically, the contributions of this paper are listed below:

- A conceptually and computationally simplified sensing method based on subband energy differences (called Max-Min ED) is developed.

- Max-Min ED and the differential method from [14] are evaluated and compared in two different sensing scenarios: (i) non-oversampled case, where the subband energy differences are due to the frequency selective channel only, and (ii) oversampled case, where the spectral variability is due to both multipath channel and the transmitted spectral shape.

- Max-Min ED methods are compared with Max/Min eigenvalue based sensing in terms of detection performance and computational complexity. It is demonstrated that both Max-Min ED methods reach similar robustness against noise uncertainty as the eigenvalue based methods have. 


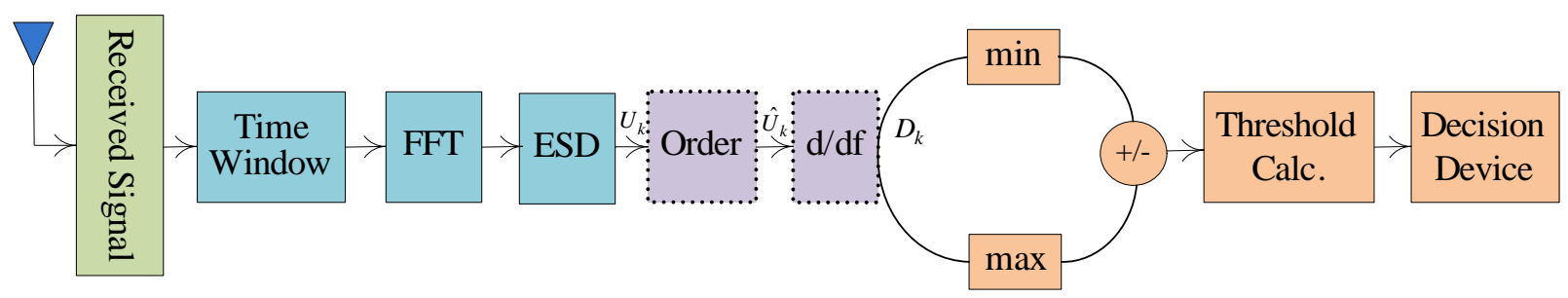

Fig. 1. Block diagram for the differential Max-Min energy detection and the proposed simplified variant (without the dotted boxes).

The remainder of the paper is organized as follows: Section II formulates first the system model and the basic ED scheme and then presents two sensing methods which are based on the variation of the subband energies. First the differential maximum-minimum energy based method from [14] is described. Then a conceptually simpler scheme, utilizing just the difference of maximum and minimum subband energies, is described. Principles for computational complexity analysis are briefly explained in Section III and numerical results for detection performance and computational complexity are given in Section IV. Closing remarks are given in Section V.

\section{System Model AND MaXimum - Minumum Energy Detector Based Spectrum Sensing}

\section{A. System Model and Problem Formulation}

Spectrum sensing is typically formulated by the following binary hypothesis testing problem [1],

$$
\begin{aligned}
& \mathcal{H}_{0}: y(n)=w(n) \\
& \mathcal{H}_{1}: y(n)=\overbrace{s(n) \otimes h(n)}^{x(n)}+w(n)
\end{aligned}
$$

where $y[n]$ is the signal observed by the sensing receiver with $s[n]$ and $w[n]$ denoting the PU information signal and the zeromean, complex, circularly symmetric, wide-sense stationary white Gaussian noise, respectively. $h[n]$ denotes the channel impulse response and $x[n]$ is the transmitted singnal. Hence, it is evident that under hypothesis $\mathcal{H}_{0}$, the PU is considered absent and $y[n]$ consists only of $w[n]$. On the contrary, under hypothesis $\mathcal{H}_{1}$ the PU information signal $x[n]$ is present along with $w[n]$. Based on this, the corresponding test statistic for the energy detector is expressed as, $T(y)=\frac{1}{N} \sum_{n=0}^{N-1}|y[n]|^{2}$, with $N$ denoting the length of the observation sequence, also referred to as sample complexity.

The decisions of the test statistics can be assumed as the Gaussian distribution due to large number of samples, which has been shown extensively to be a rather tractable and accurate assumption [12], [16]-[18]. To this end, the following formulation is straightforwardly obtained,

$$
\left.T(y)\right|_{\mathcal{H}_{0}} \sim \mathcal{N}\left(\sigma_{w}^{2}, \frac{\sigma_{w}^{4}}{N}\right)
$$

and

$$
\left.T(y)\right|_{\mathcal{H}_{1}} \sim \mathcal{N}\left(\sigma_{x}^{2}+\sigma_{w}^{2}, \frac{\left(\sigma_{x}^{2}+\sigma_{w}^{2}\right)^{2}}{N}\right)
$$

where $\sigma_{x}^{2}$ and $\sigma_{w}^{2}$ denote the variance of the PU information signal and AWGN process, respectively. Based on this and given that the instantaneous signal-to-noise ratio (SNR) is $\xi=$ $\sigma_{x}^{2} / \sigma_{w}^{2}$, the corresponding false alarm probability $\left(P_{F A}\right)$ and detection probability $\left(P_{D}\right)$ can be expressed as:

$$
P_{F A}=\operatorname{Pr}\left(T(y)>\gamma \mid \mathcal{H}_{0}\right)=Q\left(\frac{\gamma-\sigma_{w}^{2}}{\sigma_{w}^{2} / \sqrt{N}}\right)
$$

and

$$
P_{D}=\operatorname{Pr}\left(T(y)>\gamma \mid \mathcal{H}_{1}\right)=Q\left(\frac{\gamma-\sigma_{w}^{2}(1+\xi)}{\sigma_{w}^{2}(1+\xi) / \sqrt{N}}\right)
$$

respectively, where $Q(\cdot)$ is the standard Gaussian complementary cumulative distribution function and $\gamma$ denotes the predefined energy threshold.

The variance of the PU information signal is practically unknown and thus the value of $\gamma$ is typically determined by the assumed noise variance and targeted false alarm probability as $\gamma=\sigma_{w}^{2}\left(1+Q^{-1}\left(P_{F A}\right) / \sqrt{N}\right)$.

In practice, the estimation of exact noise variance is not possible. The detection and false alarm probabilities as functions of the SNR depend critically on the accuracy of noise variance estimate. In practice, the noise variance can be expected to be in the range $\sigma_{w}^{2} \in\left[(1 / \rho) \sigma_{n}^{2}, \rho \sigma_{n}^{2}\right]$ where $\rho>1$ is a parameter that quantizes the size of the uncertainty. The noise uncertainty is usually expressed in $\mathrm{dB}$ units as $x=10 \log _{10} \rho$. In the presence of noise uncertainty, the expressions for $P_{F A}$ and $P_{D}$ are modified as follows [12]

$$
\begin{array}{r}
P_{F A}=\max _{\sigma_{w}^{2} \in\left[\frac{1}{\rho} \sigma_{n}^{2}, \rho \sigma_{n}^{2}\right]} Q\left(\frac{\gamma-\sigma_{w}^{2}}{\sigma_{w}^{2} / \sqrt{N}}\right) \\
=Q\left(\frac{\gamma-\rho \sigma_{n}^{2}}{\sqrt{\left(\rho \sigma_{n}^{2}\right)^{2}}}\right) \\
P_{D}=\min _{\sigma_{w}^{2} \in\left[\frac{1}{\rho} \sigma_{n}^{2}, \rho \sigma_{n}^{2}\right]} Q\left(\frac{\gamma-\sigma_{w}^{2}(1+\xi)}{\sigma_{w}^{2}(1+\xi) / \sqrt{N}}\right) \\
=Q\left(\frac{\gamma-\sigma_{n}^{2}((1 / \rho)+\xi)}{\sqrt{\left(\sigma_{n}^{2}((1 / \rho)+\xi)\right)^{2} / N}}\right)
\end{array}
$$

B. Maximum Minumum Energy Detector Based Spectrum Sensing with or without Differentiation

This differential approach, which is seen in Fig. 1 can be separated to the following steps [13]-[15]:

- Estimating the energies across a number of subbands over the total sensing frequency band. 
- Ordering the calculated subband energies.

- Performing differentiation process for the ordered subband energies.

- Finding the maximum and minimum of the energy differentials.

- Comparing the test statistic with the threshold which is calculated according to Neyman-Person test.

1) Energy Spectral Density Approximation: The first task is to perform the $N_{F F T}$-point FFT operation on rectangularly windowed sets of $N_{F F T}$ samples. The subband signals can be expressed as:

$$
Y_{m}[k]=\left\{\begin{array}{ll}
W_{m}[k] & \mathcal{H}_{0} \\
S_{m}[k] H_{k}+W_{m}[k] & \mathcal{H}_{1}
\end{array}\right\}
$$

where $S_{m}[k]$ is the transmitted signal by primary users as it is present at the $m^{\text {th }}$ FFT output sample in subband $k$, and $W_{m}[k]$ is the corresponding channel noise sample. The subband width is assumed to be small enough such that the channel appears as flat fading within each subband and the channel frequency response can be modeled through the subband gains $H_{k}$. When the AWGN only is present, the white noise is modeled as a zero-mean Gaussian random variable with variance $\sigma_{w}^{2}$, i.e., $W_{m}[k] \sim N\left(0, \sigma_{w}^{2}\right)$. $S_{m}[k] \sim N\left(0, \sigma_{k}^{2}\right)$ where, $\sigma_{k}^{2}$ is the variance (power) at subband $k$. The subband ED process can be summarized as $U_{k}=\frac{1}{L_{t}} \sum_{m=1}^{L_{t}}\left|Y_{m}[k]\right|^{2}$ with $L_{t}=N / N_{F F T}$. At this point, with the aid of the central limit theorem, we are able to make the following approximations:

$$
U_{k}= \begin{cases}\mathcal{N}\left(\sigma_{w}^{2}, \frac{2}{L_{t}} \sigma_{w}^{4}\right) & \mathcal{H}_{0} \\ \mathcal{N}\left(\left|H_{k}\right|^{2} \sigma_{k}^{2}+\sigma_{w}^{2}, \frac{2}{L_{t}}\left(\left|H_{k}\right|^{2} \sigma_{k}^{2}+\sigma_{w}^{2}\right)^{2}\right) & \mathcal{H}_{1}\end{cases}
$$

2) Ordering: The second stage includes placing the subband energies the order of their magnitude. This step does not effect the statistical properties of of the ordered sequence, denoted as $\widehat{U}_{k}$. Thus the probability distribution of $\widehat{U}_{k}$ follows (9).

3) Differentiation: The ordered subband energy sequence is differentiated such that $D_{k}=\widehat{U}_{k+1}-\widehat{U}_{k}$. This operation can be thought of as subtracting two normally distributed random variables as shown in (9). Hence:

$D_{k} \approx \begin{cases}\mathcal{N}\left(0, \frac{4}{L_{t}} \sigma_{w}^{4}\right) & \mathcal{H}_{0} \\ \mathcal{N}\left(E\left[\widehat{U}_{k}\right]-E\left[\widehat{U}_{k-1}\right], \frac{4}{L_{t}}\left(\left|H_{k}\right|^{2} \sigma_{k}^{2}+\sigma_{w}^{2}\right)^{2}\right) & \mathcal{H}_{1}\end{cases}$

Under $\mathcal{H}_{0}$, this results in having a normal distribution with a zero mean and a variance which has twice the value of variance mentioned in (9). If the PU has white spectrum, all the subband energies follow the same statistics also under $\mathcal{H}_{1}$, and the mean becomes zero. This indicates that the method is not sensitive to the noise uncertainty.
4) Threshold Calculation and Decision Device: The decision stage comes after determining the maximum and minimum of the differences of the ordered subband energy sequence. At this stage of the algorithm, this difference between the maximum and minimum is compared with a predetermined threshold. This threshold can be obtained from the target $P_{F A}$ using the Neyman-Pearson test [19] with zero mean and double variance compared to the basic ED. According to this test, when $D_{\max }-D_{\min }>\gamma$, the signal is assumed to be present, otherwise it is expected that there is only noise in the band of interest.

We propose to consider also a simplified approach which, in the same way as the described differential scheme, utilizes the variations of the power spectrum for primary user detection. However, we remove the ordering and differentiation stages. Then the algorithm simply searches the maximum and minimum of the subband energies and uses their difference as the decision statistic. The test statistic follows the distribution of (10) under $\mathcal{H}_{0}$. Under $\mathcal{H}_{1}$, the variance is the same as in (10) but the mean becomes $E\left[U_{\max }\right]-E\left[U_{\min }\right]$.

\section{Computational Complexity Analysis of MAX-MIN ENERGY BASED SENSING}

The computational complexity is one of the most crucial parameters of spectrum sensing algorithms. Especially, complexity effects the sensing time directly and this means that the signal can be missed due to the high computational complexity. Numerical results of the traditional eigenvalue and presented Max-Min based spectrum sensing algorithms will be discussed in detail in the next section.

The main complexity of the proposed scheme comes due to the following stages:

- $N_{F F T}$-point Discrete fourier transform (DFT) introduces $O\left(N_{F F T} \log \left(N_{F F T}\right)\right)$ operations.

- Finding of the Max \& min energy values is needed and the complexity is $O\left(N_{F F T}\right)$.

In the differential version, also the ordering and differences of $N_{F F T}$ values are required, with complexity $O\left(N_{F F T}\right)$.

\section{NumericAl Results}

The detection probabilities for basic energy detector (ED), traditional Max/Min eigenvalue based detector [20], Max-Min ED with differentiator, and the proposed simplified Max-Min ED are evaluated using simulations with three different channel models (Indoor, ITU-R Vehicular A and SUI-1 channels [21]). The $1 \mathrm{~dB}$ noise uncertainty case is considered as the worst-case scenario in terms of noise variance estimation. In all the addressed cases, the time record length is 10240 complex samples and 1000 Monte Carlo simulations are applied to evaluate the detection probability reliably. The target false alarm probability is $P_{F A}=0.1$. Non-oversampled and 2x-oversampled signal models are used under $20 \mathrm{MHz}$ sensing bandwidth to see the effects of correlations (in the eigenvalue based detector case) or the PSD variability (in the Max-Min ED cases). In the non-oversampled case, the correlations and PSD variability are introduced by the multipath channel only, 
whereas in the oversampled case, they are introduced both by the channel and the shape of the transmitted spectrum. The PU signal is here modeled using filtered white Gaussian noise, using an FIR filter designed with Kaiser window. We use $N_{F F T}=\{8,32\}$ for Max-Min ED methods. In eigenvalue based sensing, the smoothing factor $L=16$, which means that the sample covariance matrix size is $16 \times 16$ in the nonoversampled case and $32 \times 32$ in the oversampled case [20]. The Vehicular A channel has 6 taps and its maximum delay spreads is about $2.5 \mu \mathrm{s}$. The Indoor channel model has 16 taps and 80 ns delay spread. SUI- 1 channel model which has 3 Ricean fading taps and $0.9 \mu s$ delay spread is used as the third channel model [21].

The simulated detection probabilities for the spectrum sensing methods under consideration are shown in Figures $2-7$.

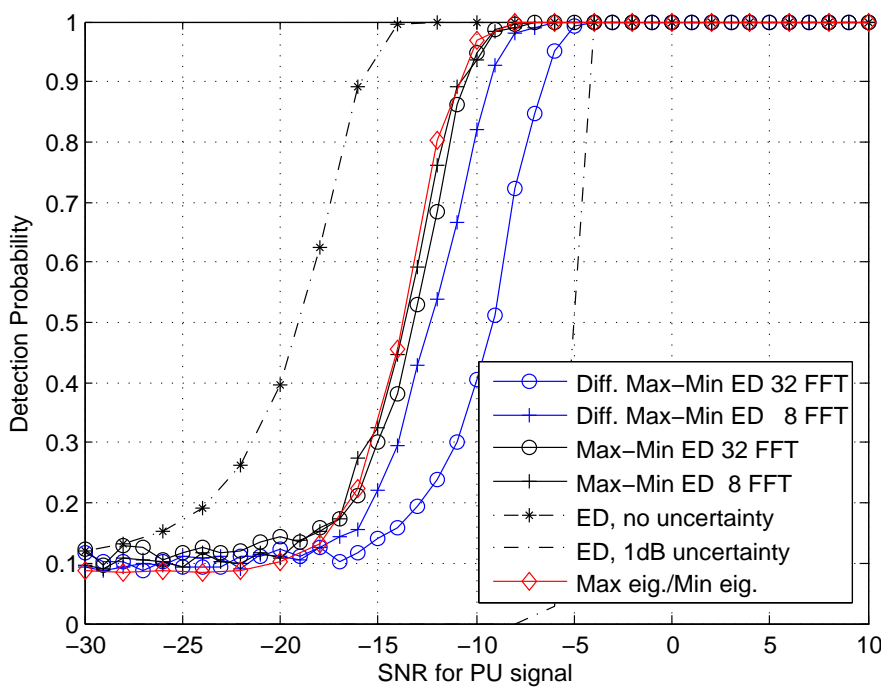

Fig. 2. Simulated detection probabilities using eigenvalue based an MaxMin energy based detectors without oversampling with 8 and 32 subchannels under Indoor channel. Theoretical performance of energy detector without noise uncertainty and with $1 \mathrm{~dB}$ noise uncertainty included as reference.

From the simulation results, we can observed that the Max-Min ED methods are able to overcome the problem of noise uncertainty in all the oversampled cases. Regarding the non-oversampled cases, these methods perform well with the Indoor and Vehicular A channels, whereas the performance with SUI-1 channel is inferior to ED with $1 \mathrm{~dB}$ noise uncertainty. The simplified Max-Min ED is competitive in detection performance with the differential Max-Min ED, with slightly improved performance in all the cases. The detection performance of the Max-Min ED methods is rather similar to that of the Max/Min eigenvalue detector. Further, it can be seen that 8 subchannels (8-point FFT) is sufficient in all the oversampled cases and also in the non-oversampled Indoor channel case. It was also verified that increasing the number of subbands beyond 32 doesn't improve the detection performance in any of the cases.

Comparison of computational complexity of the Max/Min eigenvalue detector [22] and the presented Max-Min energy based sensing methods is given in detail in Table I and

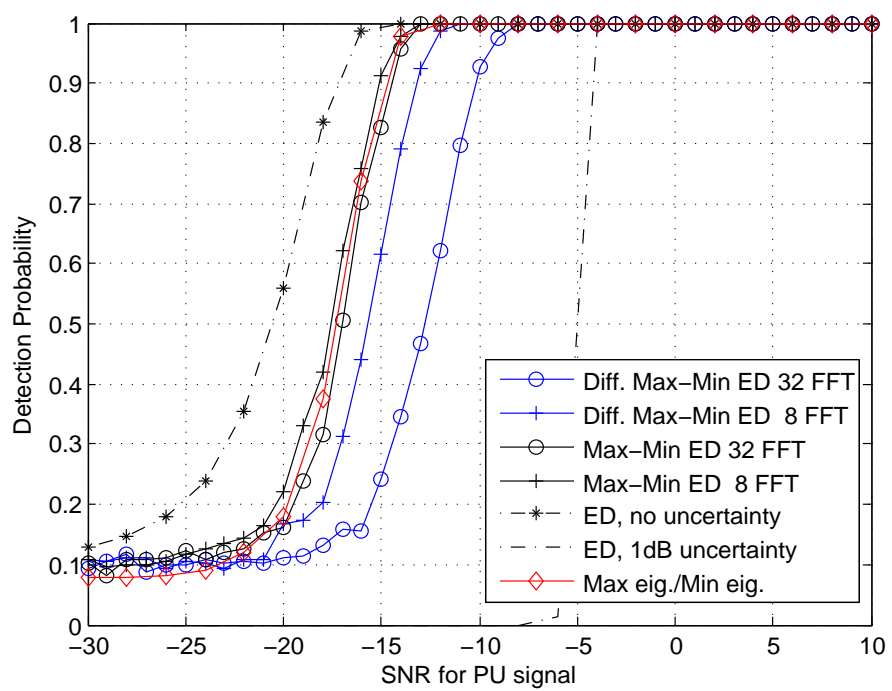

Fig. 3. Simulated detection probabilities using eigenvalue based an Max-Min energy based detectors with 2 x-oversampling, 8 and 32 subchannels under Indoor channel. Theoretical performance of energy detector without noise uncertainty and with $1 \mathrm{~dB}$ noise uncertainty included as reference.

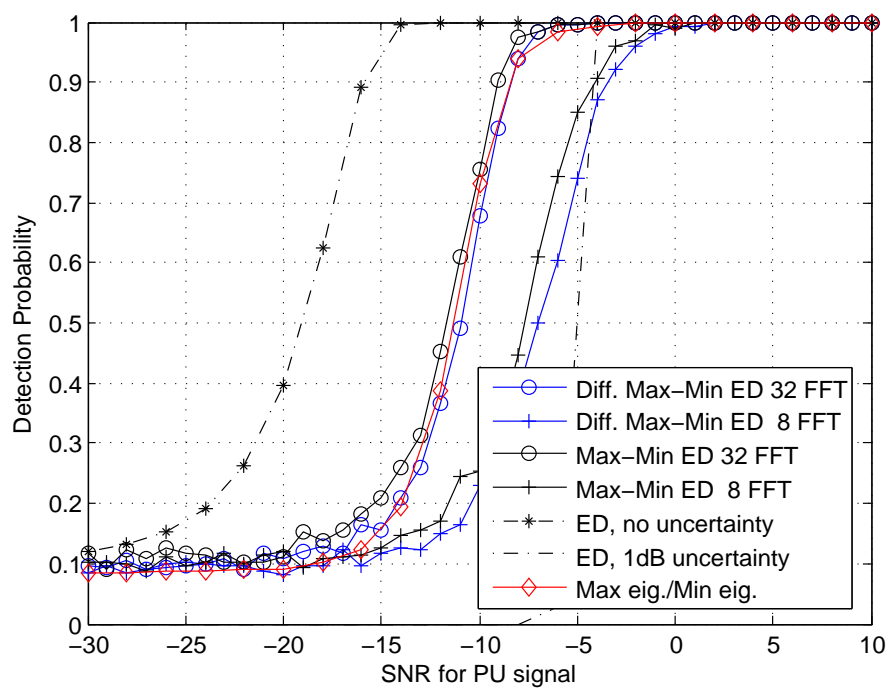

Fig. 4. Simulated detection probabilities using eigenvalue based an MaxMin energy based detectors without oversampling with 8 and 32 subchannels under ITU-R Vehicular A channel. Theoretical performance of energy detector without noise uncertainty and with $1 \mathrm{~dB}$ noise uncertainty included as reference.

Table II. While Table I shows the computational complexity for the non-oversampled case, complexity is calculated for $2 \mathrm{x}$ oversampled case in Table II.

Depending on the values of $L, M, N$ and $N_{F F T}$, significant reduction of the computational complexity can be reached. For instance from Table II, when the number of samples is 10240 with $L=16$ and $M=2$, the overall computational complexity (number of multiplications and additions) of the traditional $\mathrm{max} / \mathrm{min}$ eigenvalue based algorithm is 360448 whereas it is 122880 and 81920 under the case of $N_{F F T}=8$ for MaxMin energy with differentiation and without differentiation, respectively. Hence upon using the Max-Min algorithm, the complexity is reduced by about 78 percent. 


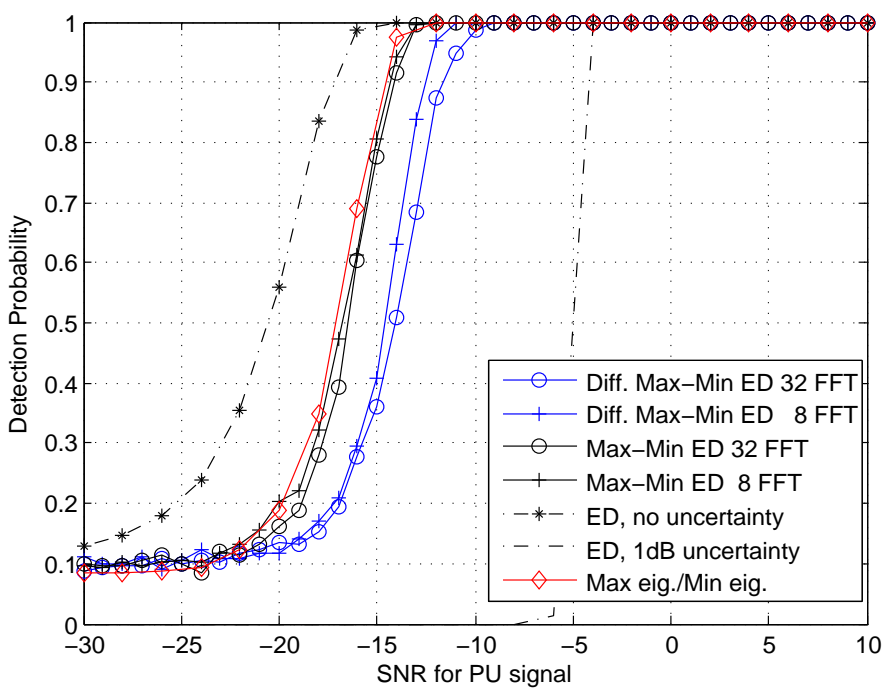

Fig. 5. Simulated detection probabilities using eigenvalue based an MaxMin energy based detectors with $2 \mathrm{x}$ oversampling, 8 and 32 subchannels under ITU-R Vehicular A channel. Theoretical performance of energy detector without noise uncertainty and with $1 \mathrm{~dB}$ noise uncertainty included as reference.

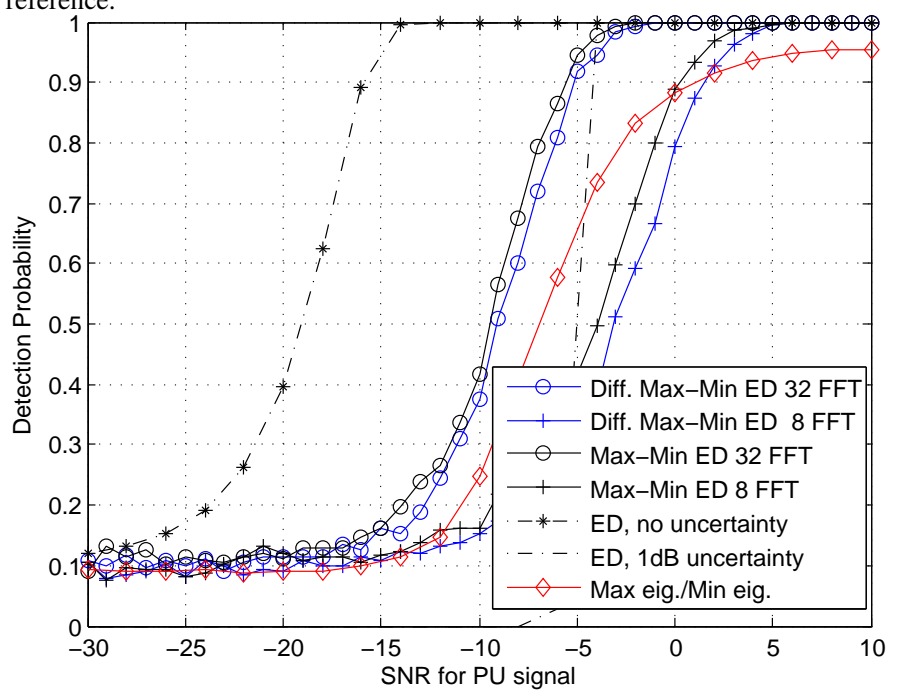

Fig. 6. Simulated detection probabilities using eigenvalue based an MaxMin energy based detectors without oversampling with 8 and 32 subchannels under SUI-1 channel. Theoretical performance of energy detector without noise uncertainty and with $1 \mathrm{~dB}$ noise uncertainty included as reference.

\section{CONCLUSION}

In this study, we investigated spectrum sensing methods which utilize the variability of the power spectral density of the received signal, which is due to multipath propagation channel and/or the shape of the transmitted PU spectrum. It was observed that the methods are able to overcome the problem of noise uncertainty in the oversampled cases and also in the non-oversampled cases if the channel exhibits sufficient frequency selectivity. In those cases, the detection performance was found to be similar to that of the Max/Min eigenvalue detector. The proposed simplified scheme reaches or exceeds the performance of the differential scheme in all oversampled cases and in non-oversampled cases with high frequency selectivity.

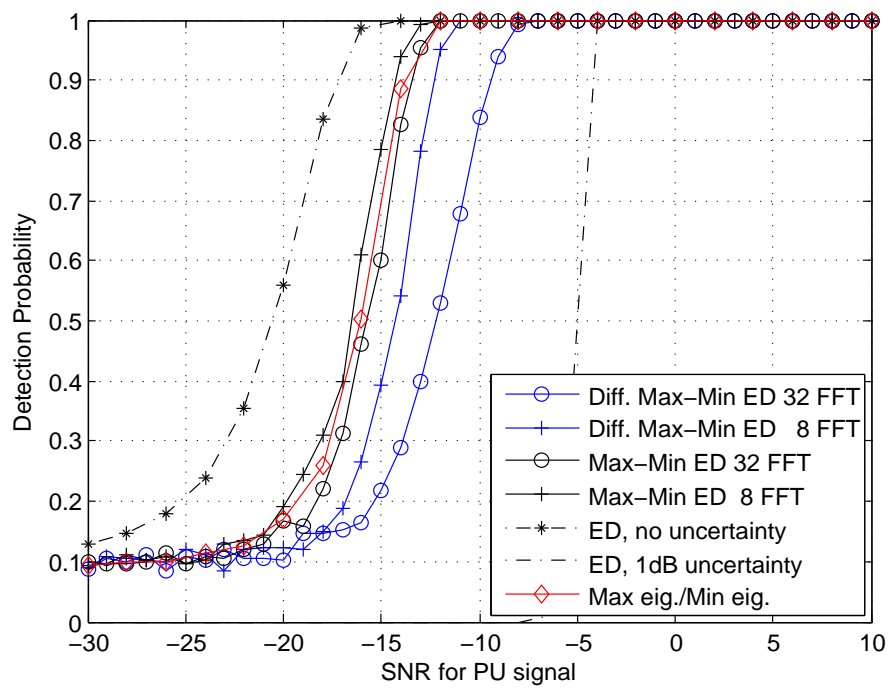

Fig. 7. Simulated detection probabilities using eigenvalue based an Max-Min energy based detectors with 2 x-oversampling, 8 and 32 subchannels under SUI-1 channel. Theoretical performance of energy detector without noise uncertainty and with $1 \mathrm{~dB}$ noise uncertainty included as reference.

While the proposed simplified scheme is conceptually quite simple compared to the differential approach, the computational complexities of these two methods are rather similar, and much smaller than the complexity of the eigenvalue based detector. The overall complexity can be reduced from $O\left((M L)^{3}\right)+M N(1+L)$ to $O\left(N_{F F T} \log \left(N_{F F T}\right)\right)+$ $O\left(N_{F F T}\right)$. The complexity reduction is due to replacing the calculation of the covariance matrix and its eigenvalues by blockwise FFT processing.

In the future work, to complete the picture, we will consider to quantify analytically the correlations introduced by the waveform and channel in different scenarios. Also other forms of decision statistic, like maximum subband energy / minimum subband energy, will be considered.

\section{ACKNOWLEDGMENT}

This work was supported in part by Tekniikan Edistmisstin (TES), GETA Graduate School, the Finnish Funding Agency for Technology and Innovation (Tekes) under the project "Enabling Methods for Dynamic Spectrum Access and Cognitive Radio (ENCOR2)" in the TRIAL Program, the Academy of Finland under the project no. 251138 "Digitally-Enhanced RF for Cognitive Radio Devices" and European Union FP7-ICT project EMPhAtiC under grant agreement no. 318362.

\section{REFERENCES}

[1] H. Urkowitz, "Energy detection of unknown deterministic signals", Proc. IEEE, vol. 55, no. 4, pp. 523-531, Apr. 1967.

[2] H. Poor, An Introduction to Signal Detection and Estimation, 2nd ed. Berlin, Germany: Springer-Verlag, 1994.

[3] S. Haykin and M. Moher, "Modern Wireless Communications", Prentice-Hall, Inc. Upper Saddle River, NJ, USA, 2004.

[4] S. Haykin, "Cognitive radio: Brain-empowered wireless communications", IEEE J. Select. Areas Commun. vol. 23, no. 2, pp. 201-220, Feb. 2005.

[5] D. Cabric, A. Tkachenko and R. Brodersen, "Spectrum sensing measurements of pilot, energy, and collaborative detection," in proc. IEEE MILCOM 2006, Washington, DC, pp. 1-7. 
TABLE I

SOME NUMERICAL VALUES OF COMPUTATIONAL COMPLEXITIES FOR SENSING METHODS (NON-OVERSAMPLED)

\begin{tabular}{|c|c|c|c|c|c|c|}
\hline & \multirow{2}{*}{ ALGORITHMS } & \multirow{2}{*}{$N_{F F T}$} & \multicolumn{4}{|c|}{ Number of samples (N) } \\
\hline & & & 512 & 1024 & 4096 & 10240 \\
\hline $\begin{array}{c}\text { Trad. } \\
\text { Alg. }\end{array}$ & $\begin{array}{c}\text { Alg. 1 } \\
\text { (max. eig./ min. eig.) } \\
\mathrm{L}=16, \mathrm{M}=1\end{array}$ & - & 12288 & 20480 & 69632 & 167936 \\
\hline \multirow{8}{*}{$\begin{array}{c}\text { Prop. } \\
\text { Alg. }\end{array}$} & \multirow{4}{*}{$\begin{array}{c}\text { MaxMin } \\
\text { (max-min energy) } \\
\text { with differentiation }\end{array}$} & 8 & 3072 & 6144 & 24576 & 61440 \\
\hline & & 16 & 3584 & 7168 & 28672 & 71680 \\
\hline & & 32 & 4096 & 8192 & 32768 & 81920 \\
\hline & & 64 & 4608 & 9216 & 36864 & 92160 \\
\hline & \multirow{4}{*}{$\begin{array}{c}\text { MaxMin } \\
\text { (max-min energy) } \\
\text { without differentiation }\end{array}$} & 8 & 2048 & 4096 & 16384 & 40960 \\
\hline & & 16 & 2560 & 5120 & 20480 & 51200 \\
\hline & & 32 & 3072 & 6144 & 24576 & 61440 \\
\hline & & 64 & 3584 & 7168 & 28672 & 71680 \\
\hline
\end{tabular}

TABLE II

SOME NUMERICAL VALUES OF COMPUTATIONAL COMPLEXITIES FOR SENSING METHODS (2X-OVERSAMPLED)

\begin{tabular}{|c|c|c|c|c|c|c|}
\hline & \multirow{2}{*}{ ALGORITHMS } & \multirow{2}{*}{$N_{F F T}$} & \multicolumn{4}{|c|}{ Number of samples $(\mathbf{N})$} \\
\hline & & & 512 & 1024 & 4096 & 10240 \\
\hline $\begin{array}{c}\text { Trad. } \\
\text { Alg. }\end{array}$ & $\begin{array}{c}\text { Alg. 1 } \\
\text { (max. eig. / min. eig.) } \\
\mathrm{L}=16, \mathrm{M}=2\end{array}$ & - & 49152 & 65536 & 163840 & 360448 \\
\hline \multirow{8}{*}{$\begin{array}{l}\text { Prop. } \\
\text { Alg. }\end{array}$} & \multirow{4}{*}{$\begin{array}{c}\text { MaxMin } \\
\text { (max-min energy) } \\
\text { with differentiation }\end{array}$} & 8 & 6144 & 12288 & 49152 & 122880 \\
\hline & & 16 & 7168 & 14336 & 57344 & 143360 \\
\hline & & 32 & 8192 & 16384 & 65536 & 163840 \\
\hline & & 64 & 9216 & 18432 & 73728 & 184320 \\
\hline & \multirow{4}{*}{$\begin{array}{c}\text { MaxMin } \\
\text { (max-min energy) } \\
\text { without differentiation }\end{array}$} & 8 & 4096 & 8192 & 32768 & 81920 \\
\hline & & 16 & 5120 & 10240 & 40960 & 102400 \\
\hline & & 32 & 6144 & 12288 & 49152 & 122880 \\
\hline & & 64 & 7168 & 14336 & 57344 & 143360 \\
\hline
\end{tabular}

[6] V. I. Kostylev, "Energy detection of signal with random amplitude", in Proc. IEEE Int. Conf. on Comm., pp. 1606-1610, May 2002.

[7] F. Digham, M.-S. Alouini and M. K. Simon, "On energy detection of unknown deterministic signals over fading channels", IEEE Trans. Commun., vol. 55, no. 1, pp. 21-24, Jan. 2007.

[8] K. Ruttik, K. Koufos and R. Jantti, "Detection of unknown signals in a fading environment", IEEE Comm. Lett., vol. 13, no. 7, pp. 498-500, July 2009.

[9] P. C. Sofotasios, E. Rebeiz, Li Zhang, T. A. Tsiftsis, D. Cabric and S. Freear, "Energy Detection-Based Spectrum Sensing over $\kappa-\mu$ and $\kappa$ $\mu$ Extreme Fading Channels", IEEE Trans. Veh. Technol., vol. 62, no.3, pp.1031-1040, March 2013.

[10] S. Atapattu, C. Tellambura and H. Jiang, "MGF Based Analysis of Area under the ROC Curve in Energy Detection", IEEE Communications Letters, vol. 15, no. 12, Dec. 2011.

[11] S. P. Herath, N. Rajatheva and C. Tellambura, "Energy detection of unknown signals in fading and diversity reception", IEEE Trans. Commun., vol. 59, no. 9, pp. 2443-2453, Sep. 2011.

[12] R. Tandra and A. Sahai, "SNR walls for signal detection", IEEE J. Sel. Topics Signal Process., vol. 2, no. 1, pp. 4-17, Feb. 2008.

[13] P. Cheraghi, Yi Ma and R. Tafazolli, "Frequency-domain differential energy detection based on extreme statistics for OFDM source sensing", in Proc. IEEE Vehicular Tech. Conf., pp. 1-5, May. 2011.

[14] P. Cheraghi, Yi Ma, L. Zhengwei and R. Tafazolli, "A novel low complexity differential energy detection for sensing OFDM sources in low SNR environment", in Proc. IEEE GLOBECOM Workshops (GC Wkshps), 2011, pp. 378-382, Dec. 2011.

[15] P. Cheraghi, Yi Ma, R. Tafazolli and L. Zhengwei, "Cluster-based differential energy detection for spectrum sensing in multi-carrier systems", IEEE Trans. Signal Process., vol. 60, no. 11, pp. 6450-6464, Dec. 2012.

[16] V. Cevher, R. Chellappa and J. McClellan, "Gaussian approximation for energy-based detection and localization in sensor networks", in Proc. IEEE SSP 2007, Madison, WI, USA, pp. 655-659.

[17] G. Taricco, "On the accuracy of the Gaussian approximation with linear cooperative spectrum sensing over Rician fading channels", IEEE Signal Process. Lett., vol. 17, no. 7, pp. 651-654, Jul. 2010.

[18] G. Taricco, "Optimization of linear cooperative spectrum sensing for cognitive radio physical layer", IEEE J. Sel. Topics Signal Process., vol. 5, no. 1, pp. 77-86, Feb. 2011.

[19] J. Neyman and E. Pearson, "On the problem of the most efficient test for statistical hypotheses", in Philosophical Trans. of the Royal Society of London, vol. 231, no.4, pp. 289-337, Feb. 1933.

[20] Y. Zeng and Y. C. Liang, "Eigenvalue-based spectrum sensing algorithms for cognitive radio "IEEE Trans. on Comm., pp. 1784-1793, June 2009.

[21] R. Jain, "Channel Models: A Tutorial", WiMAX Forum AATG, Feb., 2007.

[22] S.Dikmese, A.Gokceoglu, M.Valkama and M.Renfors, "Reduced Complexity Spectrum Sensing Based on Maximum Eigenvalue and Energy " in Proc.Wireless Communication Systems, pp. 1-5, Aug. 2013. 